\title{
LA NEUROTEOLOGÍA FUNDAMENTAL: UNA PROPUESTA PARA REPENSAR LO HUMANO
}

\author{
MONTSERRAT ESCRIBANO-CÁRCEL \\ Universitat de Valencia \\ Facultad de Teología San Vicente Ferrer, Valencia
}

\begin{abstract}
RESUMEN. Las neurociencias con su innovadora presencia nos acercan a la fisiología y arquitectura cerebral, pero también definen nuestra intimidad. Su influencia se deja sentir en la opinión pública y se expande a través de múltiples disciplinas que surgen bajo el término «neuro». Entre otras aparece la neuroteología que se sitúa en medio del conocimiento científico y la disciplina teológica. Su prioridad es describir la actividad neurocientífica correspondiente a las experiencias religiosas, meditativas o espirituales. La neurobiología permite ahora un conocimiento mayor de estas actividades y plantea cuestiones novedosas acerca del ser humano. Mi propuesta es desarrollar una neuroteología fundamental. Considero que las teologías disponen de modos racionales propios para crear pensamiento y elaborar propuestas críticas. En este artículo planteo algunas de las posibilidades que la neuroteología ofrece a las neurociencias. Por último, sostengo que la racionalidad teológica elaborada por las teologías contextuales se convierte en una fuente propositiva y disruptiva de descripciones neurocientíficas que naturalizan al ser humano.
\end{abstract}

PALABRAS CLAVE: neuroteología fundamental, racionalidad teológica, corporalidad, racionalidad neurocientífica, emergencia de la materia.

\section{Fundamental neurotheology: a proposal to rethink everything human}

\begin{abstract}
Neurosciences with its innovative presence bring us closer to the physiology and cerebral architecture, but they also define our privacy. Their influence is felt in public opinion and expands across multiple disciplines that come under the term «neuro". Neurotheology appears among others, and it is in the middle of a scientific knowledge and a theological discipline. Their priority is to describe the neuroscientist activity corresponding to the religious, meditative or spiritual experiences. Neurobiology allows a better understanding of these activities and pose novel questions about the human being. My proposal is to develop a fundamental neurotheology. Just because theologies have their own rational ways to create thinking and develop critical proposals. I raise some of the possibilities that the theological knowledge offers to the neurosciences. Finally, I believe that the theological rationale developed by the contextual theologies becomes a proactive and disruptive source of neuroscientific descriptions which naturalize the human beings
\end{abstract}

KEY WORDS: fundamental neurotheology, theological rationality, corporeality, neuroscientific rationality, emergent of the matter.

Vivimos con entusiasmo y expectación todo aquello que tiene que ver con el sistema nervioso. Tras el auge de la genética, las neurociencias son ahora una fuente constante de innovación y su irrupción es tal que influye sobre el resto de disciplinas. De modo paralelo asistimos a un despliegue tecnológico en el que la ingeniería describe con minuciosidad la actividad cerebral mientras ésta acontece. Distintas 
técnicas, como las de neuroimagen funcional fMRI, miden la funcionalidad, la espacialidad y la arquitectura de nuestros cerebros. Por primera vez, como señala Hanna Damásio, nos asomamos al interior del cerebro para comprender qué y cómo sucede la actividad neuronal ${ }^{1}$. Esta visualización más precisa nos dispone, por ejemplo, a señalar las áreas funcionales relacionadas con nuestras decisiones cotidianas, con las opciones de consumo o con las preferencias políticas. Pero al mismo tiempo, apunta también a otras funciones que en principio parecían poco relacionadas con el cerebro como son: el deseo, las pasiones, la sexualidad o los sentimientos religiosos.

Las neurociencias disfrutan de una presencia excepcional. Ejercen su influencia sobre en el resto de áreas de conocimiento, impactan en la opinión pública y transforman la comprensión de nuestra interioridad. Se extienden a través de una compleja infraestructura nutrida por enormes cantidades de dinero presupuestado mediante Estados, gobiernos, laboratorios farmacéuticos e industria tecnológica. El resultado es una institucionalización del conocimiento neurocientífico que camina pareja a la explosión de las llamadas «ciencias de la vida». Esta institucionalización está tejida por una pléyade de áreas de conocimiento reunidas bajo el prefijo neuro-, y entre ellas aparece también la neuroteología fundamental que propongo ${ }^{2}$.

\section{El Conocimiento neURocientífico. Un CONOCIMIENTO Político}

La influencia de las neurociencias se traduce en aplicaciones médicas, en métodos de intervención y en nuevas técnicas de diagnóstico que ahora afloran. Caminan de la mano de tecnologías médicas y de neurofármacos de enorme precisión Todo ello ensancha las esperanzas de millones de enfermos que padecen algún tipo de dolencia mental o de disfunción cognitiva ${ }^{3}$. Al mismo tiempo, un conocimiento mayor de nuestra arquitectura y fisiología provoca un «desplazamiento cognitivo». Es un deslizamiento que va desde el interés por la materia biológica que forma nuestro cuerpo hasta una comprensión nueva de lo humano, centrada casi exclusivamente, en los datos obtenidos en el cerebro 4 . Este "desplazamiento cognitivo», que a principios del siglo XX se situó en la biología y la psicología, desliza ahora su centro de atención hacia el cerebro.

Por ello, uno de los objetivos de este artículo es destacar que en estos momentos, las neurociencias no solo ofrecen innovación tecnológica y avances médicos, sino

1 Damásio, H., «El sueño de visualizar la función cerebral». [En línea] http://www.uoc.edu/ portal/es/sala-depremsa/actualitat/noticies/2012/noticia_143/acceptacion_es.pdf. [Consultado: 3 marzo 2016].

2 Escribano, M., Identidad y naturaleza humana desde una perspectiva neuroteológica fundamental, Tesis doctoral, Universitat de València, València, 2015.

3 Novas, C., «The Political Economy of Hope: Patients' Organizations, Science and biovalue», en: BioSocieties, 1, 2006, pp. 289-305. DOI: 10.1017/S1745855206003024; BAqué, P., «El Alzheimer, una enfermedad política», en: Le Monde Diplomatique, febrero 2016, pp. 22-24.

4 VIDAL, F., "Brainhood, anthropological figure of modernity», en: History of the Human Sciences 22, 2009, pp. 5-36. DOI: 10.1177/0952695108099133. 
también una nueva racionalidad científica. Es decir, un modo comprensivo propio desde el cual se define la intimidad y las relaciones sociales. El segundo objetivo es plantear la neuroteología fundamental como un espacio pertinente para repensar estos marcos neurocientíficos y proponer la racionalidad teológica como un modo disruptivo de pensamiento.

La racionalidad científica sitúa el punto de partida en lo que la biología neuronal manifiesta logrando así un «estilo neuromolecular de pensamiento ${ }^{5}$ » que influye sobre el resto de disciplinas y conocimientos. Desde este nuevo paradigma releemos las capacidades, los comportamientos y las creencias que se inscriben en nuestros cuerpos. Así, los datos revelados por los marcadores neuronales se traducen en el discurso legítimo que describe quiénes somos, determina las raíces de nuestras enfermedades cognitivas, anticipa nuestras decisiones de consumo e incluso localiza nuestras experiencias de fe.

Cada uno de estos verbos - describir, determinar y localizar - construye la «mirada cerebrocéntrica». A través de ella percibimos con mayor precisión la realidad material de nuestro cerebro, pero también construye una aproximación ontológica con la que anticipar y acotar quiénes seremos. Este desvelamiento del presente quiere serlo también del futuro. Así el desplazamiento cognitivo tiene grandes efectos políticos y supone también la apertura de extensos nichos de mercado que dejan paso a una posible monetización de nuestra intimidad ${ }^{6}$. Teniendo en cuenta la lógica capitalista en la que nos encontramos y la fuerza descriptiva que alcanzan las neurociencias asistimos a la elaboración de nuevas taxidermias de la población elaboradas a partir de guiones biopolíticos.

En estos momentos, el marco neurocientífico crea narraciones autorizadas acerca de nuestro comportamiento individual, social y eclesial. Naturaliza modelos que asume como universales mientras patologiza otros que señala como anormales. El resultado es que podemos estar asistiendo a un neurodeterminismo en el que las personas seamos clasificadas según determinados rasgos de nuestra vida cerebral, lo que acrecentarían aún más nuestras desigualdades sociales, políticas o económicas $^{7}$. Todo ello hace de las neurociencias una cuestión ética, política y también una preocupación teológica.

Como vemos el marco de la racionalidad neuro tiene hondas consecuencias. De ahí que debamos buscar otras racionalidades, además de la científica, que ayuden a pensar estos discursos propuestos como únicos, legítimos y universales ${ }^{8}$. Las teologías no pueden quedar al margen de estos debates.

\footnotetext{
5 Rose N., y Abi-Rached, J., Neuro. The New Brain Science and the Management of the Mind, Princeton University Press, Princeton, 2013.

6 Cooper, M., Life as surplus. Biotechnology and Capitalism in the Neoliberal era, University of Washington Press, Seattle, 2008.

7 Weber, J., «From Science and Technology to Feminist Technoscience», en: Wyer, M., BarBercheck, M., Cookmeyer, D., y Ozturk H., (eds.), Women, Science, and Technology. A reader in Feminist Science Studies, Routledge, Nueva York, 2014, pp. 543-556.

8 Un ejemplo de estas propuestas dentro de la disciplina filosófica son las investigaciones de Jesús Conill Sancho, Conill, J., «La realidad personal en perspectiva neurocientífica. La aportación zubiriana» en: Pensamiento, vol. 71, núm. 266, 2015, pp. 253-271.
} 


\section{LA RACIONALIDAD CIENTÍFICA Y SU CONSTRUCCIÓN JERÁRQUICA DE LA REALIDAD}

Las neurociencias, entre las que se incluye la neuroteología muestran, a pesar de su diversidad, objetivos comunes para desentrañar nuestra vida mental. La tecnología y la ingeniería son claves, y por primera vez, facilitan el acceso a un nivel distinto de aproximación a nuestra biología: el nivel neuromolecular. Este nuevo nivel supone un modo de medición y de comprensión que antes no teníamos. Así por una parte, las neurociencias exponen procesos bioquímicos pero, a la vez, también provocan modos propios de comprender la intimidad.

Estos sistemas de calibrado van desde las proteínas, medidas en nanómetros, hasta la totalidad del cerebro calculada en centímetros ${ }^{9}$. La variedad de niveles genera un conocimiento edificado a partir de patrones dispuestos «en cascada» que jerarquiza su comprensión. Nos encontramos con que las relaciones entre cada uno de los niveles y la influencia que ejercen los unos sobre los otros provoca, como describe la teóloga Nancey Murphy, al menos dos modos de aproximación. Uno primero que es una causalidad ascendente y otro segundo que denomina causalidad descendente ${ }^{10}$. En ellos, cada nivel inferior o superior influye sobre el resto. El resultado, como describe Arthur Peacocke, es una aproximación a la materia también jerarquizada:

«[... ] las ciencias naturales y las humanas nos dan cada vez más una imagen del mundo formada por una compleja jerarquía —o más exactamente jerarquíasde una serie de niveles de organización y de materia en las cuales cada miembro sucesivamente es un todo constituido por las partes que lo preceden en la serie» ${ }^{11}$.

La materia se presenta como un «sistema emergente» ${ }^{12}$. La cuestión de fondo es cómo entender la relación entre cada uno de sus niveles organizativos y cómo describir esta la materia emergida. La complejidad de la vida se establece a través de un ritmo de variaciones, relaciones, causalidades y restricciones. Dependiendo de cómo sean entendidas éstas abordaremos de un modo u otro la relación entre mente-cerebro, o entre los procesos mentales y fenómenos como la conciencia, la espiritualidad, la corporalidad o la sexualidad. Entonces, según establecemos la relacionalidad definimos la materia. Sin embargo, definir, interpretar y describir la materia depende y señala también nuestra comprensión del mundo. Definir la materia es mostrar la concepción de cuanto existe. Aproximarnos a sus niveles y

9 Markram H., (coord.), The Human Brain Project. A Report to the European Commission, The HBP-PS Consortium, Lausana, 2012.

10 Murphy, N., "Introduction and Overview», en: Murphy, N., Ellis, G. F. R. y O’Connor, T. (eds.), Downward Causation and the Neurobiology of Free Will, Springer-Verlag, Berlin, 2009, pp. 3-8.

11 Peacocke, A., «Emergence, Mind, and Divine Action: The Hierarchy of the Sciences in Relation to the Human Mind-Brain-Body», en: Clayton P., y Davies P., (eds.), Re-emergence of Emergence. The Emergentist Hypothesis form Science to Religion, Oxford University Press, Oxford, 2006, p. 257.

12 Deacon, T., «The Hierarchic Logic of Emergence: Untangling the Interdependence of Evolution and Self-Organization», en: Weber, B. H. y Depew, D. J. (eds.), Evolution and Learning: The Baldwin Effect Reconsidered, Massachusetts Institute Technology Press, Cambridge, 2003; MurPHY, N., y Knight, Ch. C., (eds.), Human Identity at the Intersection of Science, Technology and Religion, Ashgate, Farnaham, 2010. 
descripciones es asumir un modelo de ultimidad. No estamos, por lo tanto, ante un tema menor.

En el caso de la racionalidad neurocientífica el punto de partida son los neurodatos, es decir, algoritmos obtenidos en laboratorios. Desde ellos se describe nuestro comportamiento, la subjetividad, las creencias o la identidad sexual. Por ello, si como afirman algunos autores, las neurociencias aspiran a ser un conocimiento revolucionario que nos trasladará a un «nuevo humanismo» ${ }^{13}$ será necesario pues que revisemos los marcos, las descripciones de la materia y los modos de elaborar estos discursos ya que, como todo lenguaje científico, está siempre políticamente saturado y nunca es inocente ${ }^{14}$. Entonces cada disciplina responsablemente, entre otras también la neuroteología, debe explorar y repensar sus marcos de conocimiento a partir de los que construye el presente y el futuro.

\section{UNA NEUROTEOLOGÍA FUNDAMENTAL}

Mi interés es desarrollar el potencial crítico y propositivo que ofrece la neuroteología. Según Andrew Newberg ésta se halla en medio de las ciencias, las tradiciones religiosas y las teologías ${ }^{15}$. A pesar de su corta edad ya ha diversificado objetivos, orientaciones y metodologías. Muestra dos grandes áreas de investigación. Una primera, interesada por las experiencias del dolor y sus posibles relaciones con las creencias religiosas o espirituales. Otra segunda que pone el acento en describir la actividad neurocientífica que el cerebro refleja mientras se reviven alguna de estas experiencias. Ambas perspectivas neuroteológicas son por primera vez un estudio científico de lo religioso. Su objetivo es alcanzar una descripción neurofisiológica que sitúe y localize estas experiencias. Las consecuencias y límites que señalan son notables y amplían en mucho el estudio de lo religioso o espiritual.

Por primera vez, tenemos una disciplina, la neuroteología, elaborada a partir de estudios neurobiológicos y cuyos objetivos proyectan cómo responde nuestra vida neuronal ante creencias y experiencias de trascendencia ${ }^{16}$. Simultáneamente, se presenta la posibilidad de que podamos intervenir sobre el cerebro para paliar el dolor padecido o bien modificar la percepción de sentimientos como la rabia, la frustración o la ira y acercarlas a vivencias más creativas como son la misericordia y la reconciliación ${ }^{17}$.

13 Mora, F., Neurocultura. Una cultura basada en el cerebro, Alianza, Madrid, 2007; FrazzetTo, G., y ANKer, S., «Neuroculture», en: Nature Reviews Neuroscience 10, 2009, pp. 815-821; KURZWEIL, R., ¿Cómo crear una mente? El secreto del pensamiento humano, Lola Books, Berlín, 2013; JAR, N., "Cuando entendamos el cerebro, la humanidad se entenderá a sí misma», en: El País, 25 de mayo 2015. http://elpais.com/elpais/2015/05/21/ciencia/1432224592_729117.html [Consultado: 1 febrero $2016]$.

14 Butler, J., Marcos de guerra. Las vidas lloradas, Paidós, Barcelona, 2010, pp. 13-17; Haraway, D., «Manifesto for Cyborgs: Science, Technology, and Socialist Feminism in the 1980s», en: Socialist Review 89, 1985, pp. 65-108.

15 Newberg, A. B., Principles of Neurotheology, Asgate, Farham, 2010.

16 McNamara, P., The neuroscience of religious experience, Cambridge University Press, Cambridge, 2009.

17 RiCARD, M., «Experiencia interior y neurociencias», en: Concilium 362, 2015, pp. 13-26. 
A mi parecer, la disciplina neuroteológica no agota sus posibilidades en estas dos perspectivas anteriores. De ahí que quiera desarrollar una neuroteología fundamental situada entre las neurociencias y la tradición católica ${ }^{18}$. Como sabemos la teología es un modo peculiar de elaborar el conocimiento. Se trata de una disciplina "escandalosa» ${ }^{19}$ ya que su discurso es siempre imposible y a menudo también «indecente ${ }^{20}$. Carece de un idioma propio y ha de pedirlo prestado a otras disciplinas, de ahí que tenga un carácter interdisciplinar y secular. Pero, a mi parecer, una de las dimensiones más importantes de la racionalidad teológica es que está atravesada por una perspectiva fundamental que interroga la posibilidad y la credibilidad de su fe. Esta característica cuestiona cuáles son sus lenguajes y prácticas, y a la vez le recuerda su orientación ética hacia la promoción de la justicia social y el reconocimiento de toda vida.

Concretamente, la disciplina llamada teología fundamental describe aquello que sucede refiriéndolo a la divinidad y lo hace siempre echando mano de los esquemas sociales, económicos o sexuales disponibles. Esta cualidad fundamental la sitúa en un espacio fronterizo y epistémico interesante para cuestionar éticamente los modos en los que se produce el conocimiento tanto neurocientífico como teológico. Siendo esto así, la neuroteología fundamental se presenta como un espacio deliberativo donde repensar los modos en que las neurociencias clasifican y definen a las personas.

Mi interés es cartografiar estas propuestas y descripciones neurocientíficas desde la perspectiva que ofrece la racionalidad teológica crítica. Este modo de hacer neuroteología cuestiona, revisa y abre concepciones fundamentales sobre las que se construyen las prácticas discursivas mayoritarias o legítimas. Frente a ellas, la racionalidad teológica ofrece creativamente sus propias respuestas e introduce una perspectiva diferente para analizar la relacionalidad, la causalidad y la corporalidad humana.

En estos momentos son las teologías críticas las teologías de la liberación, las teologías feministas, las teologías queer y las teologías postcoloniales las que aportan un conocimiento más amplio de la corporalidad. El punto de partida son especialmente las mujeres y todos aquellos que son invisibilizados o considerados no-personas. Para ello recurren al esquema precartesiano que ofrecen los textos bíblicos sobre el cuerpo humano [basar/nephes]. El mundo bíblico, en su enorme variedad, entiende el cuerpo humano como un sistema integral definido por su relacionalidad, alteridad y agencia ante la trascendencia.

Estas teologías fronterizas subrayan que la vitalidad del cuerpo se percibe como un espacio abierto que genera identidades diversas, fluidas contradictorias y que siempre se muestran con un espacio político ${ }^{21}$. Una comprensión de la vitalidad

18 Escribano, M., «Neuroteología: "Un conocimiento cordialmente transformador y sinápticamente revolucionario”», en Picó, C., (ed.). Resistencia y creatividad. Ayer, hoy y mañana de las teologías feministas, Verbo Divino, Estella, 2015, pp. 161-186; «De neuroteologías y humanos. Un conocimiento disruptivo de nuestra intimidad», en: Iglesia Viva, 265, 2016, pp. 105-113.

19 WARD, G. Cities of God, Routledge, Londres,2000.

20 Althaus-Reid, M., La teología indecente: perversiones teológicas en sexo, género y política, Bellaterra, Barcelona, 2006.

21 Althaus-Reid, M., y Isherwood, L., The Sexual Theologian. Essays on Sex, God and Politics, T\&T Clark, Londres, 2004. 
nomádica de la corporalidad ${ }^{22}$ contrasta con los marcos neurocientíficos anteriores y nos ofrece al mismo tiempo la posibilidad de integrar el conocimiento neurocientífico y teológico. Una de las vías que abre es explorar una comprensión de la materia más allá de modelos jerárquicos emergentes ${ }^{23}$. A esto podrían ayudar algunos de los modelos utilizados para entender las relaciones trinitarias. Un ejemplo es el modelo de ontología relacional desarrollado por la teóloga Sarah Coakley ${ }^{24}$. Estas teologías al igual que las neurociencias comparten términos como «causalidad» y «relación», y recurren a modelos de relación. Otra posibilidad a explorar es recordar que la plasticidad, la epigénesis o la neuroregeneración suceden siempre de modo encarnado y en un contexto determinado. Considerar estas perspectivas permite inaugurar modos diversos en las que las teologías y las neurociencias pueden ir más allá del diálogo o acercamiento.

Para realizar esta propuesta crítica y propositiva la neuroteología fundamental incorpora herramientas y modos epistémicos teológicos. Dispone de una amplia trayectoria en el manejo de métodos exegéticos, lenguajes simbólicos y metáforas proféticas que le sirven para ahondar en la humanidad y en la posibilidad de la trascendencia ${ }^{25}$. Esta disciplina entiende que un mejor conocimiento funcional cerebral puede mejorar nuestra comprensión —científica y teológica- de lo humano y que descubrir cómo se construye el conocimiento neurocientífico nos permitirá elaborar modelos de ultimidad más éticos.

La disciplina teológica fundamental goza también de una dilatada tradición en cuanto al estudio de los afectos, las pasiones, el deseo, la imaginación, la voluntad o las emociones como medios para la trascendencia. Pero son ahora las neurociencias las que de nuevo cuestionan cómo sucede esto en nuestro cerebro. Sin embargo, el discurso neurocientífico a pesar de la novedad que presenta se elabora a menudo a partir de matrices heterosexistas y de concepciones heredadas que muestran sesgos de género ${ }^{26}$. Preconcepciones como éstas crean situaciones paradójicas y, como señala Sarah Coakley, exigen continuar con el análisis sobre las creencias, la racionalidad, la afectividad y el género ${ }^{27}$. Nos encontramos en los inicios de esta disciplina de ahí que debamos esforzarnos para que las neurociencias no repitan patrones neurosexistas ${ }^{28}$.

22 Braidotti, R., Lo Posthumano, Gedisa, Barcelona, 2015.

23 Juarrero, A., Dynamics in Action: Intentional Behavior as a Complex System, Massachusetts Institute Technology Press, Cambridge, 1999.

24 Conkley, S., "Epílogo: "Ontología relacional” Trinidad y ciencia», en Polkinghorne, J., La Trinidad en un mundo entrelazado, Verbo Divino, Estella, 2013.

25 Rivera, M., The Touch of Transcendence. A Postcolonial Theology of God, WJK, Londres, 2007.

26 Landström, C., "Queering Feminist Technology Studies», en WYer, M., Barbercheck, M., Cookmeyer, D., y Ozturk H., (eds.), Women, Science, and Technology. A reader in Feminist Science Studies, cit., pp. 385-399.

27 Conkley, S. God, Sexuality, and the Self. An Essay "On the Trinity», Cambridge University Press, Cambridge, 2013; The New Asceticism. Sexuality, Gender, and the Quest for God, Bloomsbury, Londres, 2015, pp. 29-100.

28 Schmitz, S., y Höppner G., (eds.), Gendered Neurocultures. Feminist and Queer Perspectives on Current Brain Discourses, Zaglossus, Viena, 2014; FInE, C., Desilusions of Gender. How Our Minds, Society, and Neurosexism Create Difference, W.W. Norton, Nueva York, 2010; Jordan-Young, R. M., Brainstorm. The flaws in the science of sex differences, Harvard University Press, Cambridge, 2010; Pitts-Taylor, V., The Brain s Body. Neuroscience and Corporeal Politics, Duke University Press, Durham, 2016. 
Al mismo tiempo, la neuroteología fundamental crítica se ve obligada a revisar su propio discurso teológico. Términos como relevación, ley natural, salvación, gracia o pecado han de ser repensados, por ejemplo, a la luz de la plasticidad que muestran nuestros cerebros. De este modo, recordar la irreductibilidad del ser humano y su neurodiversidad es una tarea compleja, pero ha de servir para experimentar otros modos de relación entre las ciencias y la teología más allá de descripciones jerárquicas o en cascada entre los distintos niveles de la materialidad.

El aparato crítico del que dispone la neuroteología fundamental le brinda inesperadas propuestas y empuja algunos de los límites de lo humano. Por todo ello, a pesar de sus debilidades epistémicas y de su invisibilidad científica, la neuroteología fundamental es una propuesta disruptiva y desestabilizadora capaz de cuestionar criterios construidos únicamente a partir de lo que nuestro cerebro manifiesta. Precisamente porque la subjetividad es inaprensible y permanece siempre abierta a posibles transformaciones que esculpen la existencia. La tarea ahora es hacer una propuesta ética que desafíe descripciones neurocientíficas que naturalizan y esencializan la vida humana reduciéndola a su biovalor ${ }^{29}$.

Universitat de València

MonTSERrat Escribano-CÁRCEL

Facultad de Teología «San Vicente Ferrer»

monescri@yahoo.es

[Artículo aprobado para publicación en diciembre de 2016]

29 Byung-Chul, H., Psicopolítica, Herder, Barcelona, 2014. 